\title{
Avaliação da infestação de piolhos mastigadores (Struthiolipeurus rheae) em emas (Rhea americana) mantidas em criadouro comercial no estado do Piauí
}

\section{Evaluation of biting lice infestations (Struthiolipeurus rhea) in rheas (Rhea americana) kept in commercial breeding in the state of Piauí}

\author{
Sandovaldo Golçalves de Moura ${ }^{1}$, Ivete Lopes de Mendonça ${ }^{2}$, Waldilleny Ribeiro \\ de Araújo Moura ${ }^{3}$, Rômulo José Vieira ${ }^{4}$, Aírton Mendes Conde Júnior ${ }^{5}$, José \\ Adalmir Torres de Souza ${ }^{6}$, Antônio Sousa Júnior ${ }^{7}$
}

\begin{abstract}
Resumo
Avaliou-se a infestação de piolhos mastigadores (Struthiolipeurus rheae) em emas (Rhea americana) mantidas em um criadouro comercial no estado do Piauí. Foram utilizadas 16 emas, sendo 12 adultas, das quais seis eram machos e seis eram fêmeas. Apenas quatro emas analisadas eram jovens. Coletaramse, aleatoriamente, duas penas das asas e duas da base do pescoço de cada animal, as quais foram colocadas em sacos plásticos fechados. Posteriormente, os piolhos foram contados, preservados em frascos com álcool etílico $70^{\circ} \mathrm{GL}$, e enviados ao Laboratório de Sanidade Animal da Universidade Federal do Piauí. Nas amostras obtidas das emas adultas, encontrou-se um total de 77 piolhos, sendo a incidência nas penas colhidas das asas, $84,4 \%$ (65), significativamente maior do que nas penas da base do pescoço, 15,6\% (12). Não foram encontrados piolhos nos animais jovens nascidos em chocadeira. As emas adultas apresentaram um alto grau de infestação por S. rheae.. A incubação artificial dos animais evitou a infestação das emas jovens por S. rheae. A alta infestação por S. rheae inviabiliza o aproveitamento comercial das penas de emas. Este é o primeiro registro da ocorrência de S. rheae em emas no estado do Piauí.

Palavras-chave: Mastigóforos. Parasitologia. Ratitas.
\end{abstract}

\footnotetext{
1 Instituto Brasileiro do Meio Ambiente e Recursos Naturais Renovavéis, Piauí, Brasil. Contato principal para correspondência: sandovaldo@yahoo.com.br.

${ }^{2}$ Universidade Federal do Piauí, 'Piauí, Brasil. E-mail: ivetemendonca@ig.com.br.

3 Universidade Federal do Piauí, Piauí, Brasil. E-mail: waldilleny@yahoo.com.br.

${ }^{4}$ Universidade Federal do Piauí, Departamento de Cirurgia e Clínica Veterinária, Piauí, Brasil. E-mail: rvieira@ufpi.br.

${ }^{5}$ Universidade Federal do Piauí, Departamento de Morfologia, Setor de Histologia e Embriologia, Piauí, Brasil. E-mail: airtoncondejr@hotmail.com.

${ }^{6}$ Universidade Federal do Piauí, Departamento de Cirurgia e Clínica Veterinária, Piauí, Brasil. E-mail: adalmir@ufpi.br.

${ }^{7}$ Universidade Federal do Piauí, Departamento de Cirurgia e Clínica Veterinária, Piauí, Brasil. E-mail: sandovaldo@yahoo.com.br.
} 


\begin{abstract}
We evaluated the level of infestation of biting lice (Struthiolipeurus rheae) in emus (Rhea americana) kept in a breeding business in the state of Piaui. We used 16 emus, 12 adults, six of them were males and six females. Only four of emus analyzed were young. Were collected randomly, two wings and two feathers of the base of the neck of each animal, which were placed in plastic bags. Later, lice were counted, preserved in jars with alcohol $70^{\circ} \mathrm{GL}$ and sent to the Laboratory of Animal Health of Federal University of Piauí for identification. In samples obtained from adult emus, met a total of 77 lice, and the incidence of the wing feathers collected, 84.4\% (65), significantly higher than in the feathers of the lower neck, $15.6 \%$ (12). No lice were found in young animals born in brooder. The adult rheas showed a high degree of infestation by S. rhea. The artificial incubation of the animals prevented the infestation of young emus S. Rhea. The high infestation S. rhea prevents the commercial use of emu feathers. This is the first record of occurrence of S. rhea in rheas in the state of Piaui.
\end{abstract}

Keywords: Mastigophores. Parasitology. Ratites.

\section{Introdução}

Os malófagos são os parasitos encontrados com maior freqüência em aves silvestres. No Brasil, poucos trabalhos têm dado ênfase à ocorrência destes em aves mantidas em cativeiro (FREITAS et al., 2002; SILVA et al., 2004).

Os piolhos mastigadores são notificados como principais responsáveis por injúrias em aves, levando à inquietação causada pela rápida movimentação e pelo hábito alimentar sobre o animal (PRICE; GRAHAM, 1997).

Amostragens rotineiras de malófagos em aves apreendidas ou cativas poderão fornecer melhor entendimento sobre a diversidade da fauna dos Phthiraptera no Brasil (VALIM et al., 2005).

Devido ao crescimento da rheacultura no Brasil e a sua importância econômica, o objetivo do presente trabalho foi avaliar a infestação de piolhos mastigadores em emas mantidas em um criadouro comercial no estado do Piauí.

\section{Material e Métodos}

A pesquisa foi desenvolvida durante o mês de abril de 2009 no criadouro comercial Condado Silvestre, registrado no Instituto Brasileiro do Meio Ambiente e dos Recursos Naturais Renováveis no Piauí (IBAMA-PI) sob o n 01/06-1309 - 963646. Esse criadouro, localiza-se na Fazenda Condado, zona rural do município de Nazária, Piauí.

Foram utilizadas 16 emas, sendo 12 adultas, seis machos e seis fêmeas, com idade entre dois e três anos, mantidas em um piquete de cinco mil metros quadrados e quatro emas jovens, com idade entre seis e oito meses, alojadas em um piquete de mil metros quadrados.

Para realizar a inspeção e avaliar a presença e distribuição dos parasitos no corpo dos animais, as emas foram capturadas pelo tratador que as segurava por trás, na base das asas, continha-as junto ao solo e em seguida uma segunda pessoa colocava um capuz em suas cabeças para que se acalmassem.

A infestação foi avaliada considerando-se informações de Ahid, Oliveira e Suassuna (2008) e os exames prévios que demonstraram a localização preferencial dos piolhos nas asas e base do pescoço. Dessa forma, coletaram-se, aleatoriamente, duas penas das asas e duas da base do pescoço dos animais experimentais.

As amostras de cada animal foram colocadas em sacos plásticos de acordo com a região de coleta, e posteriormente realizou-se a contagem dos piolhos Após a contagem, os ectoparasitos foram preservados em frascos com álcool $70^{\circ} \mathrm{GL}$ e enviados ao Laboratório de Sanidade Animal da Universidade Federal do Piauí.

No laboratório, para a identificação dos piolhos, 
realizou-se a clarificação em hidróxido de potássio $(\mathrm{KOH})$ a $10 \%$ sob temperatura ambiente. Após a lavagem em água corrente, foram diafanizados em creosoto de Faia e montados em lâmina permanente com bálsamo do Canadá. Em seguida, procedeu-se à observação em microscopia óptica em aumento de 40X, para confirmação das características taxonômicas, segundo as recomendações de Wesbroth e Seelig (1974) e Price e Graham (1997).

Para a comparação dos percentuais da presença de piolhos nas diferentes categorias, utilizou-se o teste de qui-quadrado $\left(\mathrm{x}^{2}\right)$, por meio do programa SAS System for Windows (STATISTICAL ANALYSIS SYSTEM, 1996).

\section{Resultados e Discussão}

Os piolhos encontrados nas emas examinadas foram todos Struthiolipeurus rhea (HARRISON, 1916). (Figura 1).

Figura 1 - Exemplar fêmea de Struthiolipeurus rheae, encontrado em pena da asa de Rhea americana

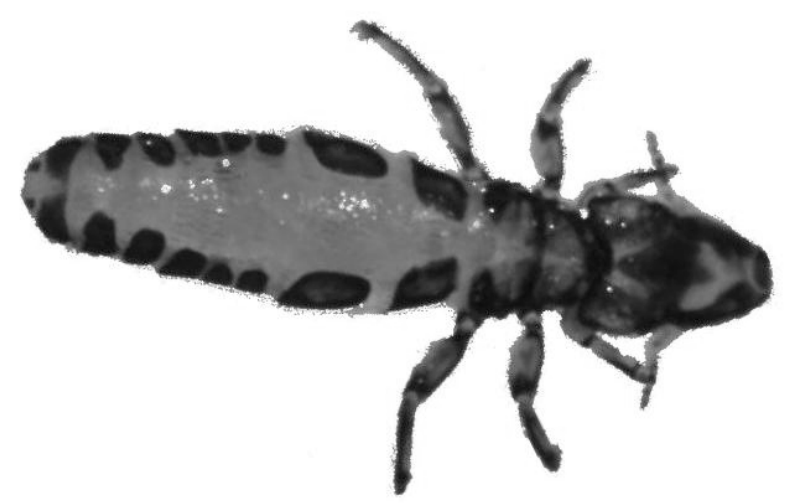

Fonte: I. L. de Mendonça, 2009.

Ao exame, verificou-se que $100 \%$ das emas adultas encontravam-se parasitadas por Struthiolipeurus rheae. A infestação de 100\% dos animais adultos é semelhante à registrada por
Ahid, Oliveira e Suassuna (2008) para S. rheae em condições cativas no estado do Rio Grande do Norte. Contudo, as emas jovens não continham piolhos; diferindo do observado pelo autor supracitado que também encontrou infestação de $100 \%$ para esta categoria animal.

No presente trabalho, a ausência de infestação nos animais jovens pode ser explicada pelo fato dos mesmos terem nascido em chocadeira, pois segundo Ahid, Oliveira e Suassuna (2008) os filhotes são infestados pelos pais logo após o nascimento.

Tabela 1 - Distribuição da freqüência do aparecimento de $S$. rheae em relação à região de coleta das penas

\begin{tabular}{lcc}
\hline Região & Ectoparasitos & Média por pena \\
\hline Asa & $65(84,4 \%)$ & 2,7 \\
Base do pescoço & $12(15,6 \%)$ & 1,0 \\
\hline
\end{tabular}

Fonte: Autores.

Analisando-se as amostras colhidas das emas adultas, encontrou-se um total de 77 piolhos, sendo a infestação das penas das asas significativamente maior $(\mathrm{p}<0,01)$ do que a das penas da base do pescoço (Tabela 1). Obteve-se uma infestação média de 2,7 piolhos por pena da asa e de 01 piolho por pena da base do pescoço. Para Sinkoc et al. (2005), a média de dois piolhos por pluma é considerada um alto grau de parasitismo.

Para Sinkoc et al. (2005), as elevadas intensidades de parasitismo podem levar, em aves destinadas à exploração de plumas, a perda da qualidade destas, acarretando prejuízo econômico para o produtor. No presente estudo, os animais parasitados encontravam-se com as penas danificadas (Figura 2), inviabilizando o aproveitamento comercial das mesmas. 
Figura 2 - Pena da asa de Rhea americana danificada pela ação de Struthiolipeurus rheae

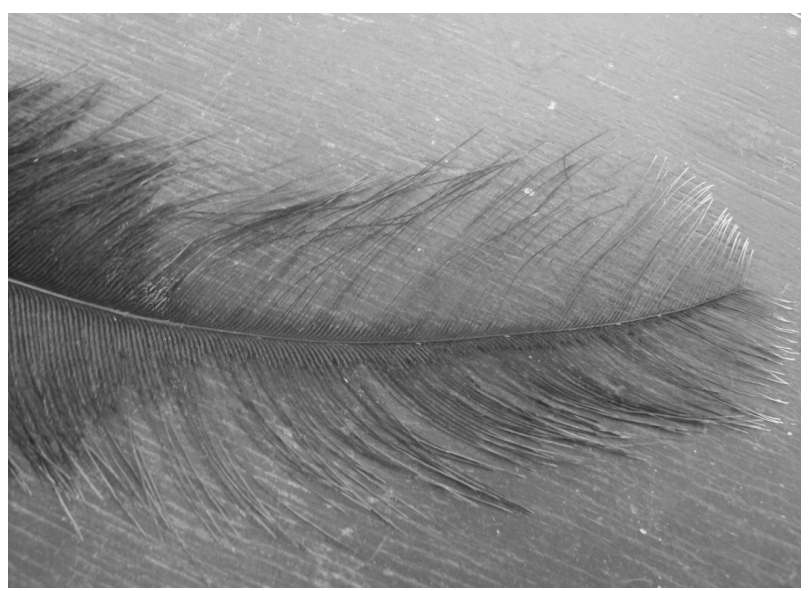

Fonte: I. L. de Mendonça, 2009.

No Brasil, cabe destacar que a ocorrência de S. rheae parasitando emas já foi registrada nos seguintes estados: Rio Grande do Norte, por Ahid, Oliveira e Suassuna (2008); Minas Gerais e Rio Grande do Sul, por Sinkoc et al. (2005) e São Paulo, por Silva et al. (2004). Esta espécie de malófago também foi encontrada parasitando avestruzes na Bahia, por Almeida et al. (2008).

No Piauí, apesar de haver quatro criadouros comerciais de emas registrados, observou-se uma ausência de informações sobre a ocorrência de S. rheae. Assim, este estudo contribuirá para que os criadores locais adotem medidas de controle e prevenção de ectoparasitos em seus planteis. Além disso, este é o primeiro registro de piolhos mastigadores da espécie Struthiolipeurus rheae (Ischnocera: Philopteridae) parasitando emas (Rhea americana) no estado do Piauí. Observou-se, neste estudo, que a incubação artificial evitou a infestação das emas jovens por S. rheae.

As emas adultas apresentaram um alto grau de infestação por S. rheae, tendo por localização preferencial as penas das asas. Como conseqüência, a alta infestação por S. rheae inviabiliza o aproveitamento comercial das penas de emas.

\section{Agradecimentos}

Ao proprietário do criadouro comercial Condado Silvestre, Sr. Natan Pinheiro de Araújo, e ao seu funcionário, Sr. Antônio Feitosa, pelo entusiasmo e apoio despendido à realização da presente pesquisa.

\section{Referências}

AHID, S. M. M.; OLIVEIRA, M. F.; SUASSUNA, A. C. D. Ectoparasitismo por Struthiolipeurus (HARRISON, 1916) (Phthiraptera: Philopteridae) em Rhea americana (Rheiformes: Rheidae) em condições cativas no município de Mossoró, Rio Grande do Norte, Brasil. Ciência Animal Brasileira, Goiânia, v. 9, n. 3, p. 767770, 2008.

ALMEIDA, M. A. O.; DUARTE, L. F. C.; ROCHA, J. S.; SILVA, M. S. A.; GUIMARÃES, J. E.; AYRES, M. C. C. Ocorrência de ectoparasitos em avestruzes (Struthio camelus) criadas no semi-árido baiano. Revista Brasileira de Parasitologia Veterinária, Jaboticabal, v. 17, n. 3, p. 155-157, 2008.

FREITAS, M. F. L; BOTELHO, M. C. N.; LEITE, A. S; MAGALHÃES, V. S; SOBRINHO, A. E.; OLIVEIRA, R. A.; OliVEIRA, M. H. C. C.; OliveIRA, J. B. Ectoparasitos de aves silvestres mantidas em cativeiro no estado de Pernambuco, Brasil. Entomologia y Vectores, Rio de Janeiro, v. 9, p. 25-33, 2002.

PRICE, M. A.; GRAHAM, O. H. Chewing and sucking lice as parasites of mammals and birds. Washington: Departament of Agriculture ARS, 1997. (Technical Bulletin, n. 1849).

SILVA, S. O.; OLIVEIRA, H. H.; FRICCIELO, R. H., SERRA-FREIRE, N. M. Malófagos parasitas de aves campestres cativas do Zoológico Municipal Quinzinho de Barros, Sorocaba (SP), Brasil. Entomologia y Vectores, Rio de Janeiro, v. 11, n. 2, p. 333-339, 2004.

SINKOC, A. L.; MULLER, G.; BRUM, J. G. W.; SOARES, M. P.; OLIVEIRA, L. T.; GONÇALVES, L. P. D. Ocorrência de Struthiolipeurus rheae (Phthiraptera: Ischnocera: Philopteridae) em Rhea americana (Rheiformes: Rheidae) no Brasil. Arquivo Instituto Biológico, São Paulo, v. 72, n. 4, p. 535-538, 2005.

STATISTICAL ANALYSIS SYSTEM. System for microsoft windows: release 6.12. Cary, 1996. CD-ROM. 
VALIM, M. P.; TEIXEIRA, R. H. F.; AMORIM, M.; SERRA-FREIRE, N. M. Malófagos (Phthiraptera) recolhidos de aves silvestres no zoológico de São Paulo, SP, Brasil. Revista Brasileira de Entomologia, Curitiba, v. 49, n. 4, p. 584-587, 2005.

WESBROTH, S. H.; SEELIG, A. W. Struthiolipeurus rheae (Mallofaga: Philopteridae), an ectoparasita of the commom rhea (Rhea americana). Journal of Parasitology, Lawrence, v. 60, n. 5, p. 892-894, 1974.

Recebido em 10 de junho de 2011

Aceito em 20 de abril de 2012 
